\title{
25 Research Square \\ Posterior deep neck infections at a single territory referral center
}

\section{Jing-Chun Zhao}

Jilin University First Hospital

\section{Nan Zhang}

The first hospital of Jilin University

Zhen-Dong Wu

The first hospital of Jilin University

Jian Wang

The first hospital of Jilin University

\section{Qing-Hua Yu}

The first hospital of Jilin University

\section{Lei Hong}

The first hospital of Jilin University

\section{Kai Shi}

The first hospital of Jilin University

Jia-Ao Yu (D 84858892@qq.com )

\section{Research article}

Keywords: Infection, neck, surgical procedures, abscess, diabetes mellitus

Posted Date: May 9th, 2019

DOl: https://doi.org/10.21203/rs.2.9534/v1

License: (1) This work is licensed under a Creative Commons Attribution 4.0 International License. Read Full License 


\section{Abstract}

Background Although the incidence and mortality of deep neck infection has decreased, this infection still relatively frequent and can be associated with lethal complications. In this study, the authors present our clinical experience of patients with posterior deep neck infection (PDNI) diagnosed and treated in a territory reconstructive unit in northeastern China. Methods A retrospective chart review of patients diagnosed with PDNI from January 2009 and December 2018 was performed. A data analysis was performed relating to demographic characteristics, clinical presentation, comorbidities, bacterial culture, laboratory and radiographic evaluations, diagnostic clues, management, complications as well as the clinical course and outcome. Results During the tenyear period there were 174 consecutive patients admitted to our reconstructive center with final diagnosis of PDNI were included. All the patients were adults with the majority were male (67.2\%). The patient mean age was 51.3 years (range, 15 - 88 years). There were 114 patients (65.5\%) who had associated systemic diseases, with the most common comorbidity was diabetes mellitus (40.2\%). Common presented clinical symptoms were pain (90.8\%), swelling (85.1\%) and erythema (77\%) of the neck. Surgical treatment was performed in all the patients and most of them (83.9\%) received the first surgery within $24 \mathrm{~h}$. The most commonly isolated pathogen was Staphylococcus aureus (30\%). Vancomycin (21.3\%) was the most commonly used antibiotics, followed by cefepime (18.4\%). All the patients survived and discharged with mean duration of hospitalization of 28.7 days. Those patients with underlying systemic diseases (31.4 \pm 12.35 days) or complications (41.0 \pm 12.5 days) tended to have a longer hospital stay. The mean cost of admission per patient was $47644 \mathrm{RMB}$. Conclusion This study highlights the high cost burden of PDNI patients. Those patients with underlying systemic diseases or complications tended to have a longer hospital stay.

\section{Introduction}

A deep neck infection (DNI) is a serious and potentially life-threatening infection, located in the potential spaces and fascial planes of the neck, which may be either abscess formation or cellulitis. The mortality rate of DNIs has decreased significantly compared with that of early reports since the improvement of modern diagnostic techniques, widespread availability of antibiotics, critical patient support modalities and early surgical intervention; however, DNIs remain to cause significant morbidity and mortality rates and present an important challenging problem [1,2].

Potential life-threatening complications can develop when diagnosis and treatment is delayed, such as upper airway obstruction, descending mediastinitis, thrombosis of the jugular vein, septic shock, pneumonia and disseminated intravascular coagulopathy [3-5].

Many etiological or predisposing factors are very important in the prevalence of DNI and modifying its outcome, which may result in death. Typically, DNIs develop in patients with diabetes mellitus (DM), immunosuppression, history of radiation and/or chemotherapy, chronic renal failure, HIV, arteriosclerosis, congenital cysts and fistulas, drug abuse or alcoholism [6,7]. Therefore, with increasing frequency of antibiotic resistance, relative frequency and their serious complications, DNIs should not be ignored, particularly in immunocompromised patients and those with low socioeconomic status [8].

Prompt recognition, timely and accurate diagnosis with laboratory and imaging tools, adequate and appropriate antimicrobial therapy, early radical surgical drainage or debridement, as well as management of complications are the keys to a successful treatment for DNIs. 
Previous retrospective analyses have been done on patients with $\mathrm{DNI}$, concentrating on the infection located in the potential spaces or fascial planes of the anterior neck. No recent information on the situation in the incidence, severity, cause(s) and occurrence of complications in posterior deep neck infection (PDNI) is available which may have their special characteristic. The main purpose of the present study was to present our own experience of the clinical course of PDNI to identify clinical features and emphasize the importance of appropriate treatment selection in those patients. The authors also compared our own findings with those recently published by other authors.

\section{Methods}

Participants All available medical records of patients treated for PDNI at the Department of Burns of the First Hospital of Jilin University (a tertiary referral academic center) in northeast China, during a 10-year period (between January 2009 and December 2018) were retrospectively investigated. Patients of all age groups and both genders were included. Only patients with abscesses or infections deeper than the superficial layer of deep cervical fascia were included in this study. The diagnosis of PDNI was based on clinical presentations and/or imaging studies [computed tomography (CT), X-ray, or neck sonography], laboratory or surgical finding. Patients with anterior neck infections, posterior superficial cellulitis or abscesses, infections due to external neck injuries (traumatic or surgical), associated with tuberculosis, and infections in head and neck malignant tumors were excluded. Those who did not complete the treatment or cases with incomplete data were also excluded.

Management of PDNI In our department, the treatment protocol had been formulated as follows: when a patient with the suspicion of PDNI was admitted, we performed thorough physical examination and peripheral blood laboratory tests. Imaging studies such as neck soft tissue radiography or neck CT scan were used selectively. A paired swab and tissue culture had been obtained for the identification of underlying microorganisms. Initial empirical intravenous antibiotics were immediately administered before the culture results were available. Thereafter, the treatment regimen and dosage of antibiotic agents were later modified based on the bacterial culture and sensitivity report.

When the diagnosis was established or highly suspected, prompt surgical debridement followed by hospital admission was performed. Wound dressings were changed every day following the surgery, or negative pressure wound therapy were changed every three to five days. For patients with signs and symptoms of severe bacteremia, sepsis or septic shock at presentation, critical patient care in intensive care unit was administered and surgery was postponed. In cases with persisting hypoalbuminemia, two-to-four intravenous administrations of $50 \mathrm{~mL}$ of $20 \%$ albumin solution were considered. Anemia was corrected with blood transfusion in critically ill patients when informed consent was obtained from the patient or their guardians.

Measured variables and data collection Data of the following clinical variables were reviewed and grouped into: a) Demographic information: age, sex, seasonal distribution, date and year of presentation; b) Predisposing factor: any underlying systemic diseases; c) Clinical features: length of the disease from the first symptoms to the date of admission, history of previous antibiotic use before hospitalization, clinical manifestation and physical examination signs, subcategory of infection (abscess or necrotizing fasciitis); d) Diagnostic procedures: clinical, laboratory data, bacteriology, and imaging studies; e) Treatment protocol, complication and outcome: antibiotic use during hospitalization, type and number of surgical procedures, length of hospitalization, and economic burden of the patients, complications, recurrence, and overall survival. 
The presence of underlying comorbidity in each patient including the patients' medical history of hypertension, DM, chronic liver disease, chronic pulmonary disease, end-stage/chronic renal disease, and coronary artery disease, were reviewed.

Clinical symptoms and signs of PDNI, including fever, neck pain, restricted movement of neck, neck swelling and neck skin fistulization, neck erythema, and localized increase in temperature, were collected.

The laboratory data were obtained from all the patients on the day of admission included peripheral blood white blood cell (WBC) count, neutrophil count, hemoglobin, albumin, blood glucose, and serum C-reactive protein (CRP). The long-term control state of DM was evaluated using the glycated hemoglobin (HbA1c) level (HbA1c:囚 $7.0 \%$, good control; $>7.0 \%$, poor control). Blood culture (anaerobic/aerobic) was performed in cases with repeated fever with body temperature greater than $38.0 \mathrm{C}$ or with signs of bacteriemia, sepsis or septic shock. Purulent samples were taken and examined in surgical patients from swabs of the wound and sent for aerobic wound cultures and antibiotic sensitivity with strict asepsis. Since the specimen collection technique prevented testing for anaerobic bacterial cultures, anaerobic cultures were not carried out in all cases. Image studies included neck soft tissue radiography or CT scan when necessary.

Two sub-categories of deep neck infections were established according to their character as previously reported [9]. Accordingly, patients with an evident abscess pocket(s) in the posterior deep cervical space were defined as the "deep cervical abscess" group, whereas those suffering from severe necrosis with soft-tissue infection spreading along the posterior deep cervical fascia and cervical muscles were defined as the "necrotizing fasciitis" group. Cervical necrotizng fasciitis was confirmed by histologic examination and operative report documentation.

Complications of PDNI identified in these patients during the clinical course were defined as sepsis, septic shock or bacteremia, that are all serious complications that justify the importance of this pathology that requiring intensive care in addition to antibiotic administration and/or initial surgical intervention during the initial hospitalization.

To evaluate if there were seasonal clustering, we defined spring as March to May, summer as June to August, fall as September to November and winter as December to February.

A recurrent disease was defined as the reappearance of infection after clinical remission.

The overall results of this study were expressed as numbers and percentages of cases for categorical variables, means \pm standard deviation (SD) and ranges of values for continuous variables. The findings were compared to those in the available literature.

\section{Results}

Patient demographics During the 10-year study period, a total of 210 patients with PDNI were identified, and thirty six cases were excluded from the study. Ultimately, a cohort of 174 eligible cases was enrolled into this study. Of them, 117 were males (67.2\%) and 57 were females (32.8\%), with a male-to-female ratio of 2.05/1. The mean age of the patients was $51.3 \pm 15.6$ years (range, $15-88$ years), with the age distribution of patients curve peaked in the fifth decade of life (42/174, $24.1 \%)$, followed by sixth decade $(39 / 174,22.4 \%)$. The age and sex distribution is shown in Fig.1. Among the 174 patients, 62 (35.6\%) were working persons, 112 (64.4\%) were nonworking (students, unemployed persons, farmers, retired people). Throughout the study period, the number of 
inpatients treated was steady during the first four years, however, it showed an upward trend in the number of presented cases since 2013, while there were four cases of PDNI in 2009, it increased to 35 cases in 2018, with peak prevalence in 2017 (43 cases). The chronological distribution of the PDNI patients is showed in Fig. 2. The number of PDNI inpatients was highest in December (21 patients) and lowest in July (9 patients) (Fig. 3). The most common season for PDNI was winter $(31.03 \%, 54 / 174)$, followed by fall $(28.74 \%, 50 / 174)$, summer $(20.69 \%$, $36 / 174)$, and spring $(19.54 \%, 34 / 174)$. PDNI was found year-round, however, winter seemed much more common. Table 1 shows the baseline demographics of all the patients.

Predisposing factor There were 114 patients (65.5\%) who had underlying systemic diseases; 81 were men, and 33 were women, with a mean age of $52.9 \pm 14.35$ years. In this patient group, the most common predisposing factor was type-2 DM in 70 patients (40.2\%), followed by hypoalbumin in 62 patients (35.6\%) and anemia in 56 patients (Table 2). Ninety five patients $(54.6 \%)$ were smokers, and 58 patients $(33.3 \%$ ) were alcoholics. There was no case of known intravenous drug abuse, or known primary or acquired immunodeficiency.

Clinical features Posterior neck abscess formation was noted in 170 patients $(97.7 \%)$ and cervical necrotizing fasciitis in only four patients (2.3\%). Each one of representative case was shown in Fig. 4 and Fig. 5.

The mean time from symptom onset to the demand for health services was $11.0 \pm 6.9$ days (range, 1-30 days). The most common presenting symptoms on admission were neck pain (158 cases, $90.8 \%$ ), followed by local neck swelling and had a progressive increase in size in few last days, and neck erythema, documented in 148 (85.1\%) and $134(77 \%)$ patients, respectively.

The mean body temperature at presentation was $37.2 \pm 0.79^{\circ} \mathrm{C}$ (range, $\left.36.5-39.6^{\circ} \mathrm{C}\right)$. At admission, physical examination revealed that 7 patients $(4.0 \%)$ had signs of bacteremia or septic shock at the time of diagnosis, and those collected blood cultures. A summary of the clinical features of the patient cohort are presented in Table 3 .

\section{Diagnostic procedure}

Laboratory findings Regarding the laboratory test findings, the mean $\pm S D$ values at the time of admission were: WBC count $=13.18 \pm 8.08 \times 10^{9} / \mathrm{L}$ (range, $1.87-39.95 \times 10^{9} / \mathrm{L}$ ) and neutrophils count $=10.88 \pm 7.71 \times 10^{9} / \mathrm{L}$ (range 1.14-29.29 $\times 10^{9} / \mathrm{L}$ ) in all the 174 cases with available data, respectively. In addition, leukocytosis (WBC $>11.0 \times 10^{9} / \mathrm{L}$ ) was detected in 54 patients $(31.0 \%)$, and leukopenia $\left(\mathrm{WBC}<4.0 \times 10^{9} / \mathrm{L}\right)$ in six patients $(3.4 \%)$. Twenty four patients $\left(13.8 \%\right.$ ) had a WBC count of more than $20.0 \times 10^{9} / \mathrm{L}$. Hemoglobin $=119.3 \pm 23.66 \mathrm{~g} / \mathrm{L}$ (range, $50-179 \mathrm{~g} / \mathrm{L}$ ) in all the 174 cases with available data, with a lower than $120 \mathrm{~g} / \mathrm{L}$ in male or $110 \mathrm{~g} / \mathrm{L}$ in female was found in 56 patients $(32.2 \%$ ). Serum albumin $=29.70 \pm 7.12 \mathrm{~g} / \mathrm{L}$ (range, $12.7-44.2 \mathrm{~g} / \mathrm{L}$ ) in 166 of 174 cases with

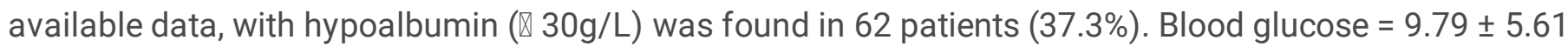
$\mathrm{mmol} / \mathrm{L}$ (range, $3.66-28.38 \mathrm{mmol} / \mathrm{L}$ ) in 170 of 174 cases with available data, with an elevation of blood glucose $(>11.1 \mathrm{mmol} / \mathrm{L}$ ) in 40 patients $(23.5 \%$ ). Glycated hemoglobin= $10.06 \pm 2.72 \%$ (range, $5.2-14.5 \%$ ) in 76 of 174

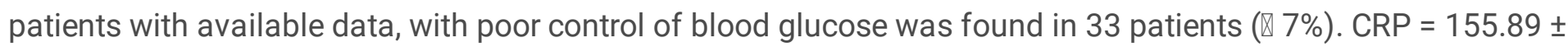
$116.10 \mathrm{mg} / \mathrm{L}$ (range, $2.7-525.0 \mathrm{mg} / \mathrm{L}$ ) in 146 of 174 cases with available data, with 68 cases $(46.6 \%)$ had the elevation of CRP level $(>3.5 \mathrm{mg} / \mathrm{L})$.

Blood cultures were routinely checked for patients with signs of sepsis, resulting positive cultures in 7 patients, with the most prevalent occurrence of Klebsiella pneumoniae (2 cases). Laboratory data at admission was presented in Table 4. 
Bacteriology The results of bacterial cultures were available for 150 of the 174 cases who underwent surgical treatment or wound swabs (86.2\%). Bacteriological samples were missing in 24 patients. There were 111 of those patients $(74 \%)$ had identifiable bacterial growths, and the remaining 39 cultures showed no bacterial growth (26\%). The most commonly isolated organisms of the positive culture were Staphylococcus aureus [ $\mathrm{n}=45,30 \%$; including 4 methicillin-resistant $S$. aureus (MRSA)], followed by Escherichia coli $(\mathrm{n}=15,10 \%)$ and Klebsiella pneumoniae ( $\mathrm{n}=11,7.3 \%)$. Polymicrobial culture was detected in 13 patients $(8.7 \%, 2-3$ species). The microbiology findings are summarized in Table 5.

Imaging findings In the current study, only a few patients underwent imaging: 21 cases (12.1\%) had ultrasound scanning, 4 cases $(2.3 \%)$ underwent neck soft tissue x-ray, and 6 patients (3.4\%) underwent CT scan. None of the patient underwent magnetic resonance imaging or other imaging examination.

Treatment protocol, complication and outcome Thirty four out of 174 patients (76.5\%) had been previously treated before admission, 16 patients (9.2\%) underwent surgical intervention combined with antibiotic therapy in regional hospital, and 18 patients (10.3\%) were already treated with antibiotics alone, orally or intravenously, in regional hospital, clinics or by themselves. Notably, thirteen patients (7.5\%) used traditional Chinese medicine topically themselves prior to their first visit to our institution.

After admission, all the patients initially received broad-spectrum intravenous antimicrobial therapy empirically at their presentation, in order to cover the majority of aerobic, gram-negative and gram-positive organisms involved in PDNIs. The antimicrobial agents and their doses were then adjusted according to microbiological bacterial culture and clinical response.

The most common treatment regimens of empirical antibiotics were vancomycin $(n=38,21.3 \%)$, followed by cefepime $(n=32,18.4 \%)$ and meropenem $(n=31,17.8 \%)$, cefuroxime $(n=26,14.9 \%)$, ceftriaxone $(n=19,10.9 \%)$ and ornidazole $(n=14,8.0 \%)$. Other antibiotics used in this study group were as follows: levofloxacin $(n=8,4.6 \%)$, cefoperazone tazobactam $(n=6,3.4 \%)$, tigecycline $(n=5,2.9 \%)$, imipenem $(n=4,2.3 \%)$, mezlocillin sodium and sulbactam sodium $(n=4,2.3 \%)$, teicoplanin $(n=3,1.7 \%)$, moxifloxacin $(n=3,1.7 \%)$, penicillin sodium $(n=2,1.1 \%)$, ceftezole $(n=1,0.6 \%)$, flucloxacillin sodium $(n=1,0.6 \%)$, sulfoxil sodium $(n=1,0.6 \%)$, cephalosporin $(n=1,0.6 \%)$ and doxycycline $(n=1,0.6 \%)$.

In this study, all the patients underwent aggressive surgery, including debridement of necrotic tissue, primary suture and drainage, and secondary skin grafting. Most of the patients (146/174, 83.9\%) received the first surgery within $24 \mathrm{~h}$. The mean time intervals from hospital admission until surgery was 1 day (range, 0-5 days). Seventy six patients (43.7\%) were subjected to a series of surgeries (2-4 operations).

In addition, supportive medical treatments was also initiated when required, which included fluid therapy, analgesics, nutritional support, blood transfusion, control of blood glucose, and antipyretics. When patients with decompensated systemic diseases were involved, appropriate specialists, mostly endocrinologist, cardiologist or internal medicine specialists, were consulted regarding the therapy.

Life-threatening complications were developed in seven cases (4.0\%), including bacteremia in four cases and septic shock in three cases. All the patients were discharged in stable condition, with the mean length of stay was $28.7 \pm 12.95$ days (range, 12-63 days). None of our patient died of PDNI or its complication. Those patients with underlying systemic diseases (31.4 \pm 12.35 days) or complications (41.0 \pm 12.5 days) tended to have a longer 
hospital stay. The mean cost of admission per patient was 47644 RMB (range, 5848 - 303579 RMB). Signs of cellulitis in two patients and abscess in one patient were found and they were hospitalized again for management.

\section{Discussion}

To our knowledge, this 10-year study of 174 patients is the only study with largest outcomes analysis of PDNI which adds to the literature of DNI by looking at the characteristics of an Asian population in northeast China. Men with PDNIs were predominant in our stud (117/174, 67.2\%), which is similar to previous reports [10,11]. Higher incidence of this infection was found among patients aged between 50 to 59 years (24.1\%), with a mean age of years 51.3 years, which is in keeping with the findings of previous study [8]. A major increase in PDNI incidence in our setting was noted in the last few years. If we consider the earlier (2009-2013) and later (20142018) periods separately, the number of patient suffering from PDNIs increase significantly in the later period (earlier: 20 patients, later: 154 patients). The reason for these discrepancies remains unclear.

From the cases that yielded positive bacterial culture in the current series, the most common microorganism identified was Staphylococcus aureus (45,30\%), with positive cultures of polymicrobial infection were identified in 13 patients (8.7\%). This result is consistent with a previous study [12]. In light of the variable pathological organisms and the rising incidence of polymicrobial infections, broad-spectrum empirical antimicrobial therapy is advocated in treating PDNIs [3,13].

Our series was consistent with previous study in that a predominance of the infections in winter [14]. However, no differences were found in the seasonal distribution of neck infection was also reported [15].

Although DNI was a kind of bacterial infection, fever was present in $64.4 \%$, and $31 \%$ had WBC count of over 11 000 cells/mm3 in the present study. Neither fever nor leukocytosis is constant findings in DNIs patients as in other report [16]. An explanation for this situation may be the use of antibiotic and anti-inflammatory treatments prior to hospitalization, and the administration of empirical broad spectrum antibiotics before obtaining culture sample, which might have masked the presentations of DNIs without fever or leukocytosis [17].

Development of modern imaging tools has greatly facilitated the diagnosis of PDNI. Computed tomography is the appropriate examination for further localized lesions and is sometimes considered compulsory for the evaluation of DNIs [18]. However, this represents only 50 percent of the positive predictive value of CT scans [19]. Ultrasound is a superior method for the investigation of superficially located lesions [16]. However囚ultrasound cannot always identify small or deep abscess and cannot provide the exact anatomical information necessary for surgical intervention [20]. Magnetic resonance imaging is also helpful in the diagnosis of PDNI, but is not commonly preferred in imaging deep neck infection as in our patients because it is expensive, not easily available, time consuming, and require good patient compliance, that may be sometimes a limitation to its use in emergency settings [21]. Surprisingly, only a relatively small number of patients in our sample underwent imaging. The reason for this discrepancy may be we diagnose the PDNI mainly depend on the clinical presentation, laboratory results and findings intraoperatively. Emergent surgery debridement was performed immediately after admission to the hospital, which is why only a small number of patients were scanned. However, we agree that imaging should always be carried out if there is any suspicion of the spread of infection towards other areas. 
The results of pus cultures from either surgery or wound swab were available in 150 of 174 patients (86.2\%); positive report of culture and polymicrobial growth was identified in $74 \%$ and $8.7 \%$, respectively. The results of positive rate were relatively lower in the present series. The present results may be explained by the use of antibiotics before admission, the high dose of empirical antibiotics before surgical intervention and, possibly, an improper sample collection may have affected the results of microbiological tests of this study. When pus drainage was available, Huang et al. [22] suggest Gram stain and acid-fast staining of pus, instead of bacterial culture alone, as early as possible before initiating antimicrobial therapy, may be helpful for pathogens identified.

Our report has shown association of PDNI with DM in 70 cases (40.2\%), which is same as compared with the study of Gujrathi et al. (36.30\%) [10]. In DM patients, hyperglycemia may impair several mechanisms of immune defense which result in predisposition to infection and complications, including impaired neutrophil function (e.g. chemotaxis, bacterial killing, phagocytosis and impaired adherence), impaired myeloperoxidase activity and antioxidant system, micro and macroangiopathies, a decrease in cytokine response during leukotriene release in humoral immunity [23-25]. In return, lipopolysaccharide secretion by bacteria and infection mediated upregulation of cytokine synthesis worsen diabetes [26]. Diabetes is a commonly recognized risk factor among the systemic disease that associated with the development of DNIs [27]. Patients with DM may exhibit a significantly higher complication rate, longer length of hospital stay, and many more concurrently infected spaces than those without DM $[4,11,28,29]$. On the other hand, previous studies also indicate that DM is not a factor in the elongated hospital stay and not necessarily a prognostic factor of DNI [30]. The impact of DM on DNI, thus, remains need to be further investigated [5].

There was a close association between DM patients and Klebsiella pneumoniae in previous studies [4, 29, 31, 32], while only six DM patients were involved in our patients (3.4\%), which was similar to those of Mejzlik et al. [33]. In that study, Klebsiella pneumonia was found in only a few cases (1.2\%) in their study. For those with DM, aggressive control of blood glucose concentration in plasma plays an important role in treating patients with diabetes with DNI [34]. Hirasawa et al. [5] recommend that in patients with DM, preventing aggravation by strictly controlling acute-phase glycemia to $250 \mathrm{mg} / \mathrm{dl}$ is necessary.

According to our experience, patients with PDNIs need to be treated as soon as possible surgically. In our study, all the patients underwent surgical treatment. Previous studies have reported that surgical treatment was used in $79-100 \%$ of patients with DNI $[10,15,16,34]$. The high proportion of surgical rate may be our hospital was a tertiary center which receive and treat patients with severe DNI. In addition, only severe cases were enrolled in this study. Patients with severe DNI were more likely underwent surgical interventions [25]. Undoubtedly, antibiotic treatment is indispensable in treatment of severe PDNIs, too.

There are several limitations to our study. First, as a retrospective study in nature in a single medical center, standardized documentation relating to the clinical patient evaluation and overall fitness status are hardly to reach. Some data on physical examinations and laboratory results were missing which may exert a great influence on the clinical course and outcome. Second, we could not eliminate our institutional bias, and there was no standardization or protocol to determine which patients should undergo immediate surgery. The timing of surgical intervention may have been influenced by an individual surgeon decision, and level of surgeon experience not captured herein. Third, this study spans with ten years with a heterogeneous patient population which may differ in infected defect and severity. Finally, skin grafting was performed in certain patients in this cases series; however, the information of donor site was not collected. Our study can only reflect experiences from 
a single medical center. However, the characteristics of PDNIs can still be deduced from our data. Further multicenter, prospective studies assessing the different characteristics of PDNIs would be helpful to overcome these limitations.

\section{Conclusions}

Patients with DM are susceptible to PDNI. When dealing with PDNIs in a high-risk group (older patients with DM or other underlying systemic diseases), more attention should be paid to the prevention of complications and shorter the length of hospital stay. Timely visit of patients is crucial for optimal treatment and warrant a better outcome, avoiding the complication and reduce the financial cost, which is of huge benefit for people lived in economically underdeveloped areas, like most of patients in our study group.

\section{Declarations}

\section{Abbreviations}

PDNI: posterior deep neck infection; DNI: deep neck infection; DM: diabetes mellitus; CT: computed tomography; WBC: white blood cell; CRP: C-reactive protein; HbA1c: glycated hemoglobin (HbA1c); SD: standard deviation.

\section{Acknowledgements}

Not applicable.

\section{Funding}

The authors have no funding to declare in relation to the content of this article.

\section{Availability of data and materials}

The datasets used and analyzed in the current study are available from the corresponding author on reasonable request.

\section{Authors' contributions}

ZJC and ZN, substantial contributions to conception and design, acquisition of data, drafting the manuscript, and final approval; WZD, WJ, YQH and LH, acquisition of data, or analysis and interpretation of data; SK and YJA, critical revision of manuscript and final approval. All authors approved the final draft prior to submission. 


\section{Consent for publication}

Informed written consent for publication of their clinical details and/or clinical images was obtained from the patient/guardian/ relative of the patient.

\section{Competing interests}

The authors declare that they have no competing interests.

\section{References}

1. Brito TP, Hazboun IM, Fernandes FL, Bento LR, Zappelini CEM, Chone CT, et al. Deepneck abscesses: study of 101 cases. Braz J Otorhinolaryngol. 2017; 83(3):341- 8.

2. Hurley RH, Douglas CM, Montgomery J, Clark LJ. The hidden cost of deep neck space infections .Ann R Coll Surg Engl. 2018; 100(2):129-34.

3. Marioni G, Staffieri A, Parisi S, Marchese-Ragona R, Zuccon A, Staffieri C, et al.

Rational diagnostic and therapeutic management of deep neck infections: analysis of 233consecutive cases. Ann Otol Rhinol Laryngol. 2010; 119(3):181-7.

4. Huang TT, Tseng FY, Liu TC, Hsu CJ, Chen YS. Deep neck infection in diabetic patients: comparisonof clinical picture and outcomes with nondiabetic patients. Otolaryngol Head Neck 2005; 132(6):943-7.

5. Hirasawa K, Tsukahara K, Motohashi R, Endo M, Sato H, Ueda Y, et al. Deep neck cellulitis: limitations of conservative treatment with antibiotics. Acta Otolaryngol. 2017; 137(1):86-9.

6. Adoviča A, Veidere L, Ronis M, Sumeraga G. Deep neck infections: review of 263 cases. Otolaryngol Pol. 2017; 71(5):37-42.

7. Santos Gorjón P, Blanco Pérez P, Morales Martín AC, Del Pozo de Dios JC, Estévez Alonso S, Calle de la Cabanillas MI. Deepneck infection. Review of 286 cases. Acta Otorrinolaringol Esp. 2012; 63(1):31-41.

8. Huang TT, Liu TC, Chen PR, Tseng FY, Yeh TH, Chen YS. Deep neck infection: analysisof 185 cases. Head Neck. 2004; 26(10):854-60.

9. Park MJ, Kim JW, Kim Y, Lee YS, Roh JL, Choi SH, et al. Initial Nutritional Status and Clinical Outcomes in Patients with Deep Neck Infection. Clin Exp Otorhinolaryngol. 2018; 11(4):293-300.

10. Gujrathi AB, Ambulgekar V, Kathait P. Deep neck space infection - A retrospective study of 270 cases at tertiary care center. World J Otorhinolaryngol Head Neck Surg. 2016; 2(4):208-13.

11. Wang LF, Tai CF, Kuo WR, Chien CY. Predisposing factors of complicated deep neck infections: $12-$ year experience at a single institution. J Otolaryngol Head Neck Surg. 2010; 39(4):335-41.

12. Bakir S, Tanriverdi MH, Gün R, Yorgancilar AE, Yildirim M, Tekbaş G, et al. Deep neckspace infections: retrospective review of 173 cases. Am J Otolaryngol. 2012; 33(1):56-63.

13. Huang TT, Tseng FY, Yeh TH, Hsu CJ, Chen YS. Factors affecting the bacteriology of deep neck infection: A retrospective study of 128 patients. Acta Otolaryngol. 2006; 126(4):396-401. 
14. Grisaru-Soen G, Komisar O, Aizenstein O, Soudack M, Schwartz D, Paret Retropharyngeal and parapharyngeal abscess in children-epidemiology, clinical features and treatment. Int J Pediatr Otorhinolaryngol. 2010; 74(9):1016-20.

15. Côrte FC, Firmino-Machado J, Moura CP, Spratley J, Santos M. Acute pediatric neck infections: Outcomesin a seven-year series. Int J Pediatr Otorhinolaryngol. 2017; 99:128-

16. Eftekharian A, Roozbahany NA, Vaezeafshar R, Narimani N. Deep neck infections: a retrospective review of 112 cases. Eur Arch Otorhinolaryngol. 2009; 266(2):273-7.

17. Bottin R, Marioni G, Rinaldi R, Boninsegna M, Salvadori L, Staffieri A. Deep neck infection: a present-day complication. A retrospective review of 83 cases (1998-2001). Eur Arch Otorhinolaryngol. 2003; 260(10):5769.

18. Wang B, Gao BL, Xu GP, Xiang C. Images of deep neck space infection and the clinical significance. Acta Radiol. 2014; 55:945-51.

19. Chuang SY, Lin HT, Wen YS, Hsu FJ. Pitfalls of CT for deep neck abscess imaging assessment: a retrospective review of 162 cases. B-ENT. 2013; 9(1):45-52.

20. Smith JL 2nd, Hsu JM, Chang J. Predicting deep neck space abscess using computed tomography. Am J Otolaryngol. 2006; 27(4):244-7.

21. Stalfors J, Adielsson A, Ebenfelt A, Nethander G, Westin T. Deep neck space infections remain a surgical challenge. A study of 72 patients. Acta Otolaryngol. 2004; 124(10):1191-6.

22. Huang CM, Huang FL, Chien YL, Chen PY. Deepneck infections in children. J Microbiol Immunol Infect. 2017; 50(5):627-33.

23. Hostetter MK. Handicaps to host defense: effects of hyperglycemia on C3 and Candida albicans. Diabetes. 1990; 39(3):271-5.

24. Chang GH, Ding MC, Yang YH, Lin YH, Liu CY, Lin MH, et al. High Risk of Deep Neck Infection in Patients with Type 1 Diabetes Mellitus: A Nationwide Population- Based Cohort Study. J Clin Med. 2018; 7(11). pii: E385. doi: $10.3390 / \mathrm{jcm} 7110385$.

25. Lin HT, Tsai CS, Chen YL, Liang JG. Influenceof diabetes mellitus on deep neck infection. J Laryngol Otol. 2006; 120(8):650-4.

26. Li X, Kolltveit KM, Tronstad L, Olsen I. Systemic diseases caused by oral infection. Clin Microbiol Rev. 2000; 13(4):547-58.

27. Wang LF, Kuo WR, Tsai SM, Huang KJ. Characterizations of life-threatening deep cervical space infections: a review of one hundred ninety-six cases. Am J Otolaryngol. 2003; 24(2):111-7.

28. Lee JK, Kim HD, Lim SC. Predisposing factors of complicated deep neck infection: an analysis of 158 cases. Yonsei Med J. 2007; 48(1):55-62.

29. Chen MK, Wen YS, Chang CC, Lee HS, Huang MT, Hsiao HC. Deep neck infections in diabetic patients. Am J Otolaryngol. 2000; 21(3):169-73.

30. Ando T, Sato M, Tabuchi T. Deep neck abscess extended to the mediastinum. Jibi to Rinsho. 1992; 38(3):21419.

31. Lin RH, Huang CC, Tsou YA, Lin CD, Tsai MH, Chen JH, et al. Correlation between imagingcharacteristics and microbiology in patients with deep neck infections: a retrospective review of one hundred sixty-one cases. Surg Infect (Larchmt). 2014; 15(6):794-9. 
32. Lee YQ, Kanagalingam J. Bacteriology of deep neck abscesses: A retrospective review of 96 consecutive cases. Singapore Med J. 2011; 52(5):351-5.

33. Mejzlik J, Celakovsky P, Tucek L, Kotulek M, Vrbacky A, Matousek P, et al. Univariateand multivariate models for the prediction of life-threatening complications in 586 cases of deep neck space infections: retrospective multi- institutional study. J Laryngol Otol. 2017; 131(9):779- 84.

34. Parhiscar A1, Har-El G. Deep neck abscess: a retrospective review of 210 cases. Ann Otol Rhinol Laryngol. $2001 ; 110(11): 1051-4$.

\section{Tables}

Table 1 Patient demographics $(\mathrm{n}=174) *$

\begin{tabular}{ll}
\hline Demographic & Results \\
\hline Sex & \\
$\quad$ Male & $117(67.2 \%)$ \\
$\quad$ Female & $57(32.8 \%)$ \\
Age (years) & $51.3 \pm 15.6$ \\
Smoker & $95(54.6 \%)$ \\
Chronic drinker & $58(33.3 \%)$ \\
Days before admission & $11.0 \pm 6.9$ \\
Antibiotics prior to admission & $18(10.3)$ \\
Traditional Chinese medicine prior to admission & $13(7.5)$ \\
Length of hospital stay (days) & $28.7 \pm 12.95$ \\
Cost (RMB) & 47644 \\
Death during treatment & 0 \\
\hline
\end{tabular}

*Data presented as mean \pm standard deviation or number (percentage, \%).

Table 2 Summarizes the comorbidities seen in the study population $(n=174$, given patient could have more than one comorbidity). 


\begin{tabular}{lc}
\hline Comorbidity & Number of patients (\%) \\
\hline None & $60(34.5)$ \\
Diabetes mellitus & $70(40.2)$ \\
Hypertension & $19(10.9)$ \\
Pneumonia & $9(5.2)$ \\
Cardiovascular diseases & $9(5.2)$ \\
Liver diseases & $6(3.4)$ \\
Renal insufficiency & $5(2.9)$ \\
Hematological diseases & $3(1.7)$ \\
Anemia & $56(32.2)$ \\
Hypoalbumin & $62(35.6)$ \\
Autoimmune disease & $2(1.1)$ \\
Psychiatric disorders & $1(0.6)$ \\
Gout & $2(1.1)$ \\
Ulcerative colitis & $1(0.6)$ \\
Upper gastrointestinal hemorrhage & $1(0.6)$ \\
Congestive heart failure & $1(0.6)$ \\
\hline
\end{tabular}

Table 3 Patients presenting characteristics of posterior deep neck infection $(n=174$, given patient might present more than one symptom/sign)

\begin{tabular}{lc}
\hline Clinical characteristics & Number of cases (\%) \\
\hline Neck pain & $158(90.8)$ \\
Neck swelling & $148(85.1)$ \\
Neck erythema & $134(77.0)$ \\
Localized increase in temperature & $128(73.6)$ \\
Fever & $112(64.4)$ \\
Restricted neck movement & $86(49.4)$ \\
Neck skin fistulization & $52(29.9)$ \\
Inadequate incision and drainage of neck abscess & $16(9.2)$ \\
\hline
\end{tabular}


Table 4 Laboratory data at admission

\begin{tabular}{lc}
\hline Laboratory parameters (reference range) & Mean values \\
\hline White blood cells count $\left(\times 10^{9} / \mathrm{L}, 3.5-9.5\right)$ & $13.18 \pm 8.08$ \\
Neutrophil count $\left(\times 10^{9} / \mathrm{L}, 1.8-6.3\right)$ & $10.88 \pm 7.71$ \\
C reactive protein $(\mathrm{mg} / \mathrm{L}, 0-3.5)$ & $155.89 \pm 116.10$ \\
Blood glucose (mmol/L, 4.1-5.9) & $9.79 \pm 5.61$ \\
Glycated hemoglobin $(\%, 4.27-6.07)$ & $10.06 \pm 2.72$ \\
Hemoglobin (g/L, $115-150)$ & $119.3 \pm 23.66$ \\
Serum albumin (g/L, $40-55)$ & $29.70 \pm 7.12$ \\
Blood culture (case) & 2 \\
Klebsiella pneumoniae & 1 \\
Streptococcus viridans+Escherichia coli & 1 \\
Enterococcus & 1 \\
Methicillin-resistant S. aureus & 1 \\
Staphylococcus aureus & \\
No growth & \\
\hline
\end{tabular}

Table 5 Bacteriology in patients with posterior deep neck infection $(n=150)$. 


\begin{tabular}{|c|c|c|}
\hline Microorganisms & & Number of cases (\%) \\
\hline \multicolumn{3}{|l|}{ Gram-positive } \\
\hline & Staphylococcus aureus & $45^{*}(30)$ \\
\hline & Streptococcus viridans & $3(2)$ \\
\hline & Coagulase-negative Staphylococci & $4(2.7)$ \\
\hline & Streptococcus pyogenes & $2(1.3)$ \\
\hline & Enterococcus & $3(2)$ \\
\hline & Streptococcus anginosus & $4(2.7)$ \\
\hline & Staphylococcus haemolyticus & $1(0.7)$ \\
\hline & Micrococcus Kristinae & $1(0.7)$ \\
\hline & Staphylococcus epidermidis & $1(0.7)$ \\
\hline & Streptococcus constellatus & $1(0.7)$ \\
\hline & Streptococcus agalactiae & $1(0.7)$ \\
\hline \multicolumn{3}{|l|}{ Gram-negative } \\
\hline & Escherichia coli & $15(10)$ \\
\hline & Klebsiella pneumoniae & $11(7.3)$ \\
\hline & Pseudomonas aeruginosa & $3(2)$ \\
\hline & Enterobacter cloacae & $9(6)$ \\
\hline & Corynebacterium & $1(0.7)$ \\
\hline & Actinobacillus & $1(0.7)$ \\
\hline & Acinetobacter baumannii & $2(1.3)$ \\
\hline & Proteus mirabilis & $1(0.7)$ \\
\hline & Stenotrophomonas maltophilia & $1(0.7)$ \\
\hline & Enterobacter aerogenes & $1(0.7)$ \\
\hline Mixed flora ${ }^{\#}$ & & 13 (8.7) \\
\hline No growth & & $39(26)$ \\
\hline Total & & $150(100)$ \\
\hline
\end{tabular}

* Includes 4 cases of methicillin-resistant $S$. aureus.

\# Includes Streptococcus anginosus, Escherichia coli, Pseudomonas aeruginosa, coagulase-negative Staphylococci, Streptococcus viridans, Staphylococcus aureus, Enterococcus, Klebsiella pneumoniae, Enterobacter cloacae, Acinetobacter baumannii, Proteus mirabilis.

\section{Figures}




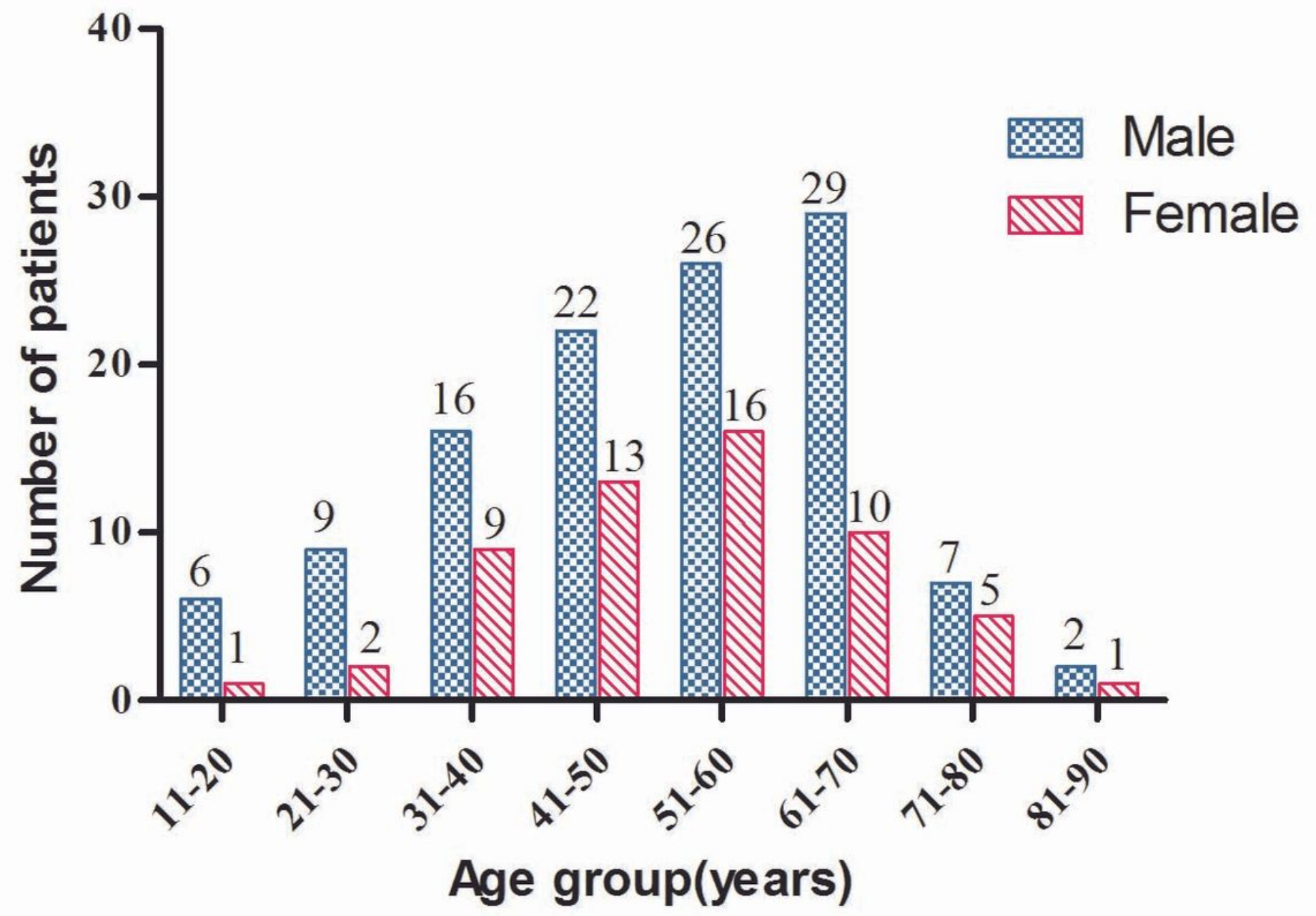

Figure 1

Age and sex distribution of patients. 


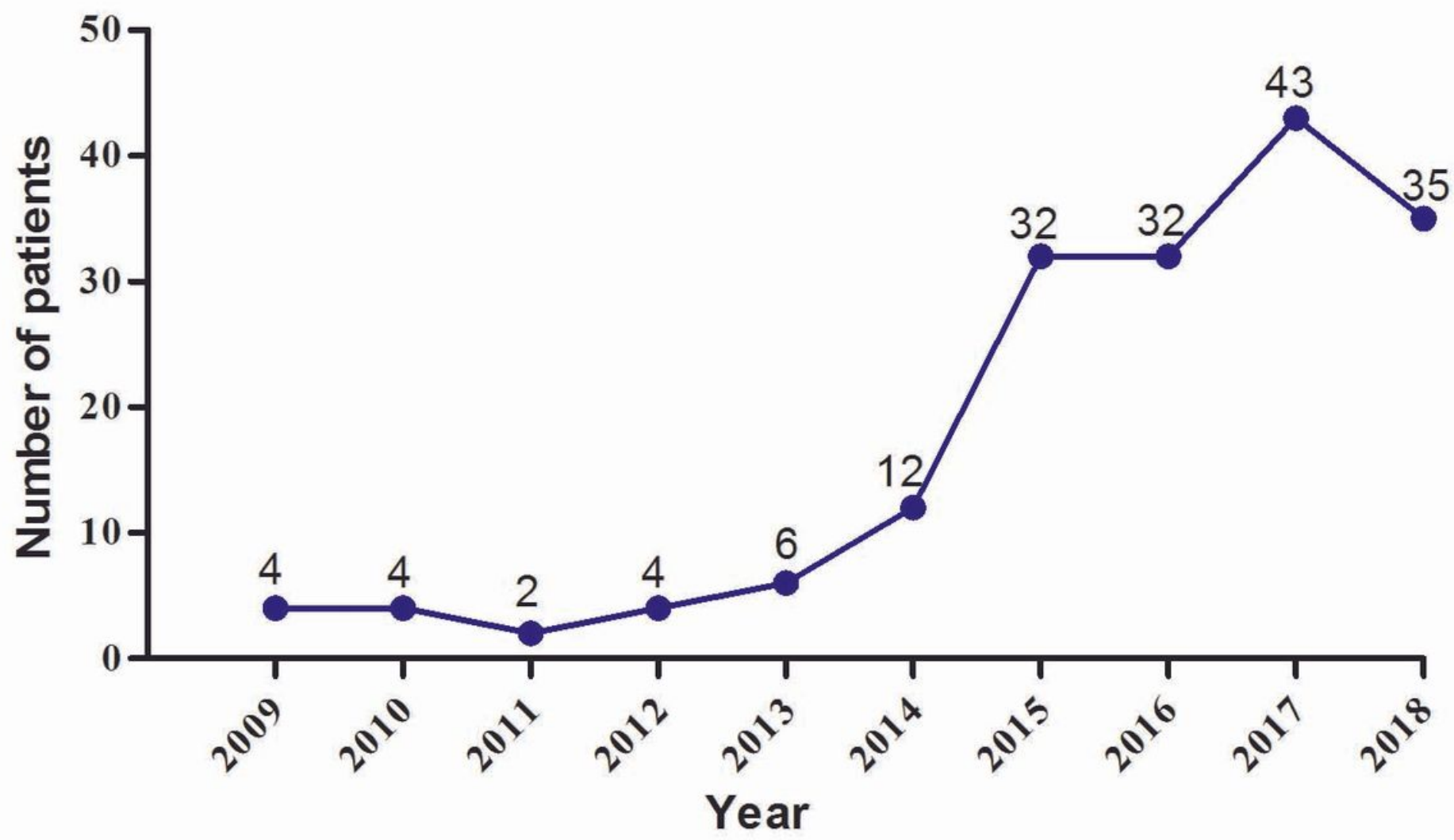

Figure 2

Annual distribution of posterior deep neck infections. 


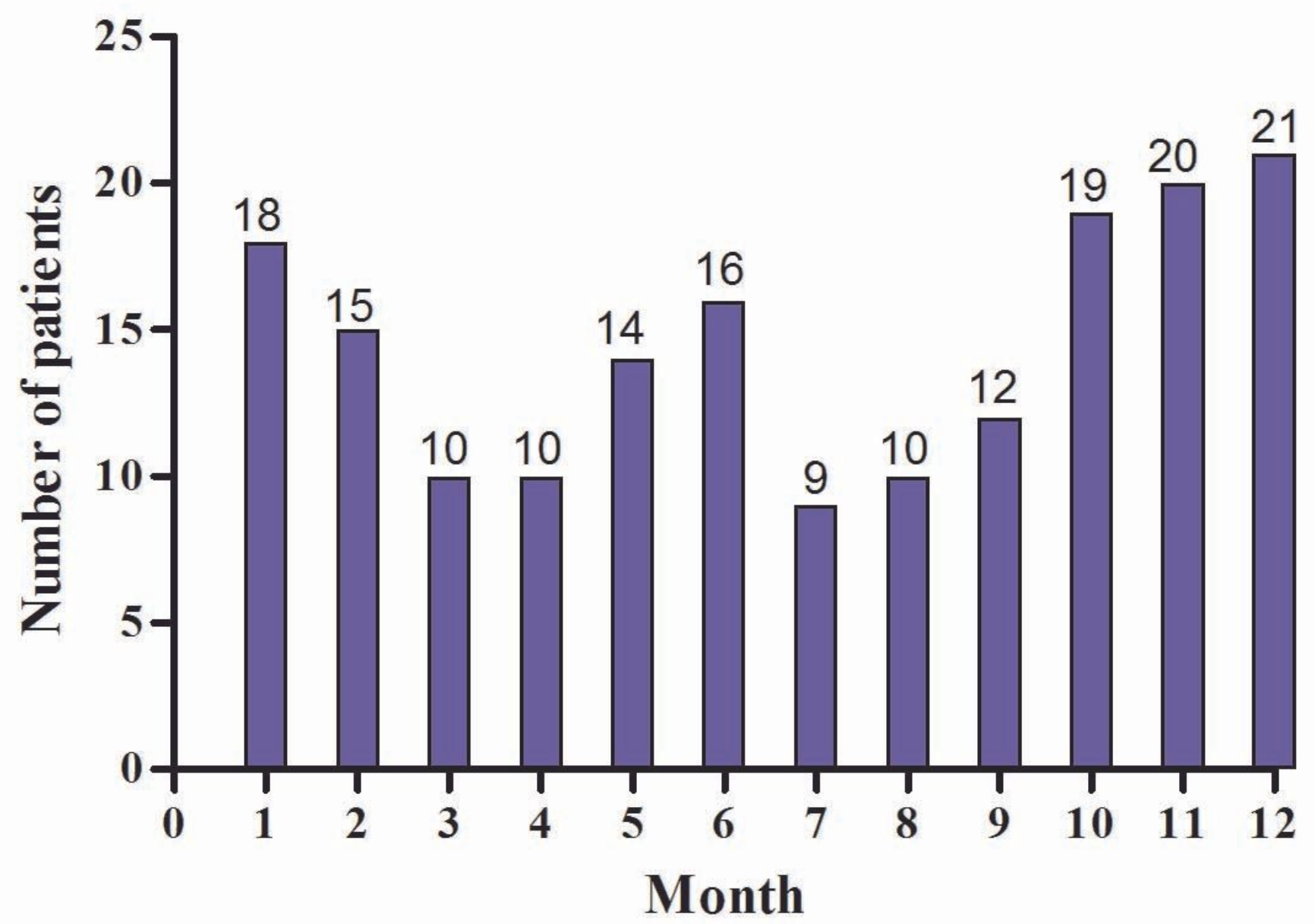

Figure 3

Distribution of the patients throughout the years (month distribution). 

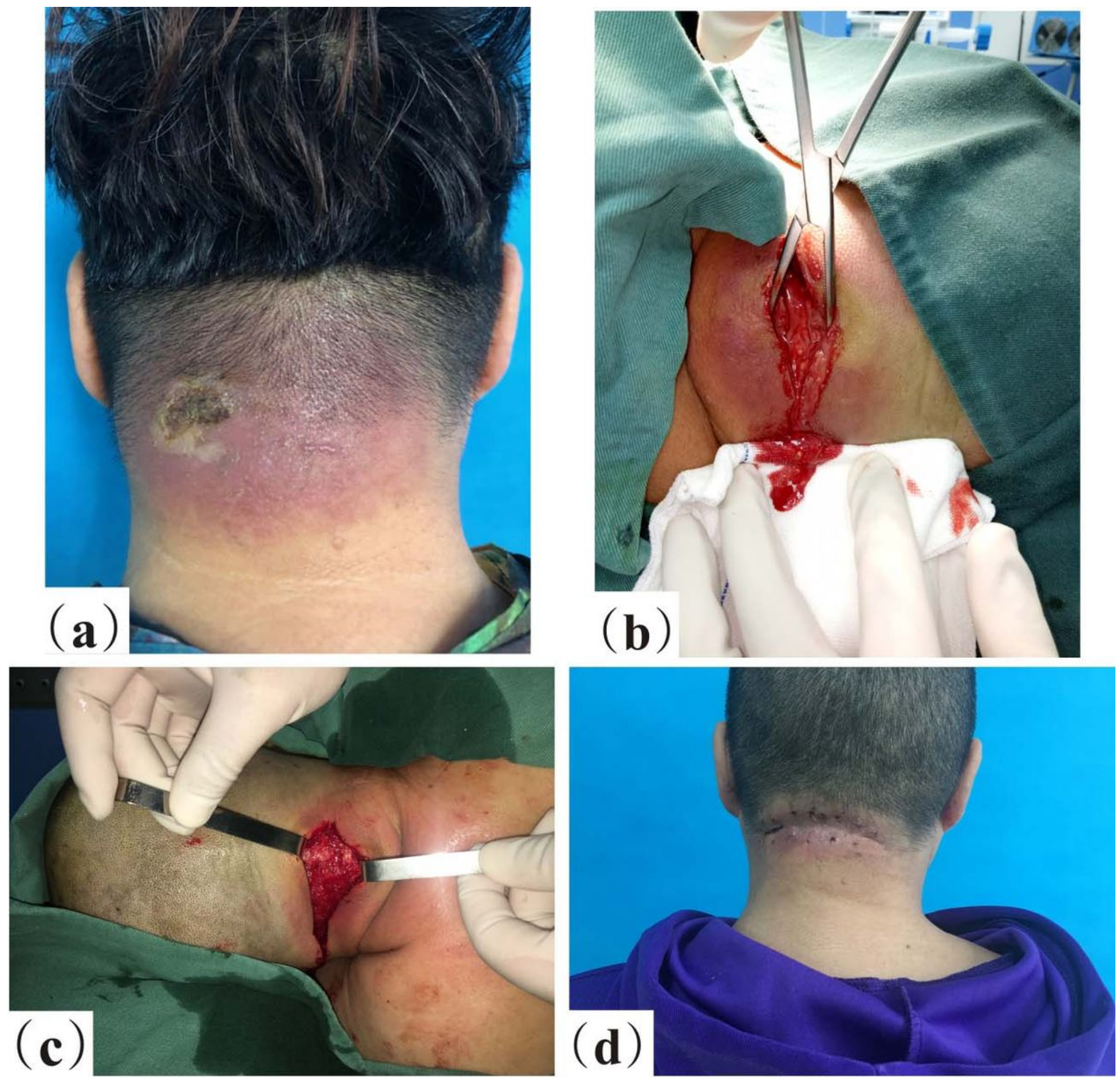

\section{Figure 4}

A 58-year-old female with a history of diabetes mellitus, who was afebrile with hard, erythematous and hot swelling in the posterior region of the neck with spontaneous drainage of purulent fluid without showing crepitus. (a): Picture of wound on admission. (b): Incision and drainage was performed immediately. (c): Surgical wound after complete debridement. (d) Final outcome after suture of the wound. The incision healed well. 

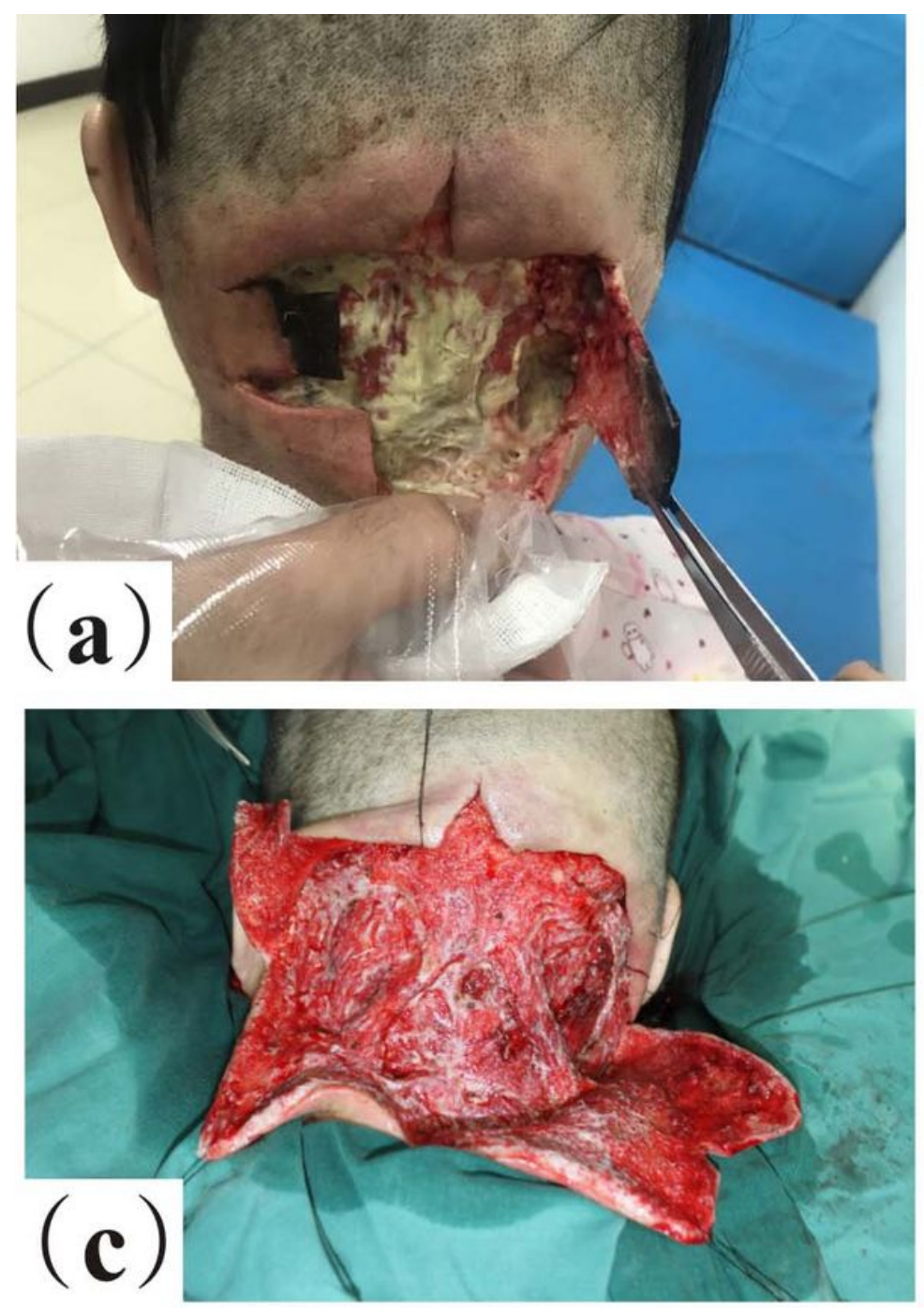
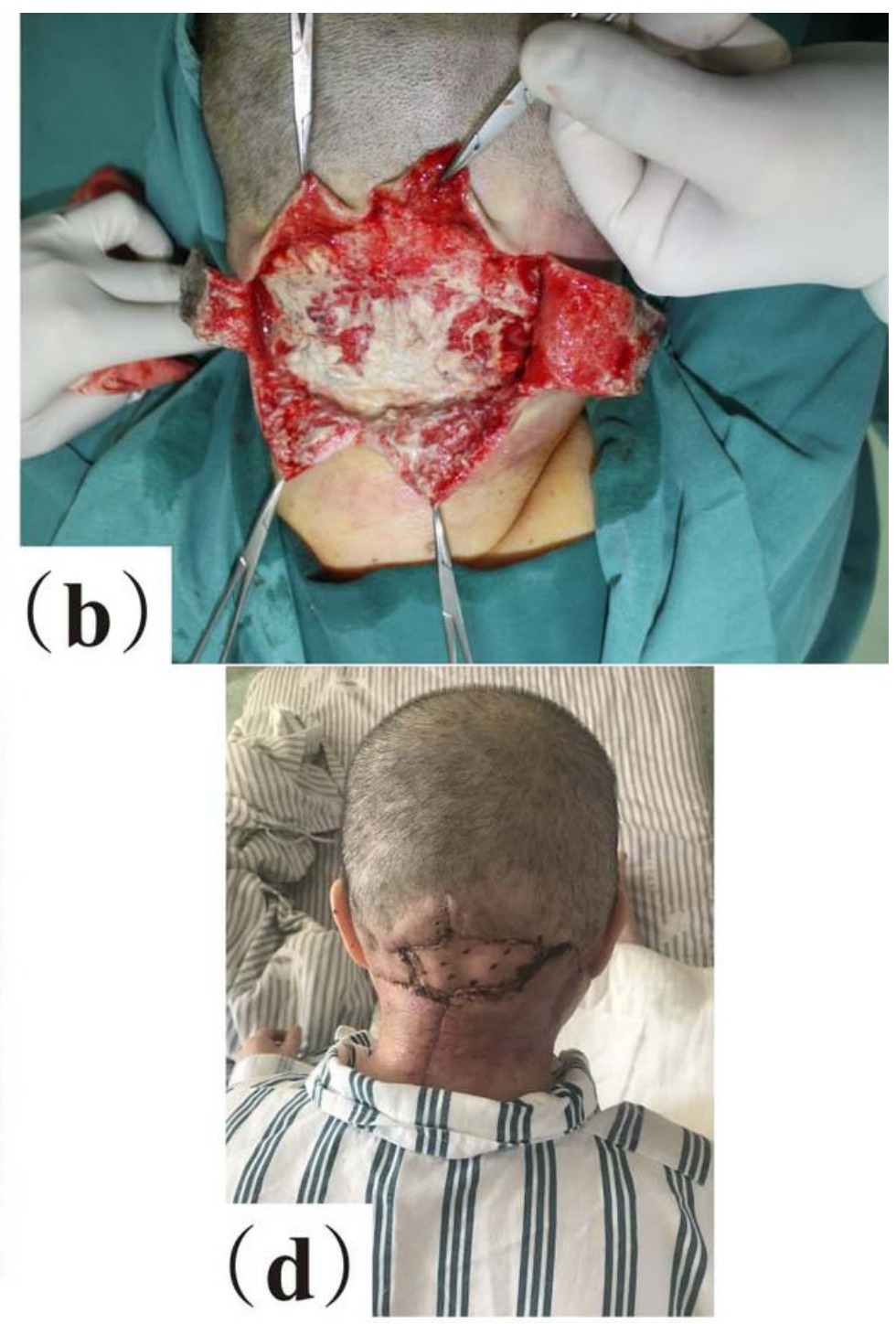

Figure 5

A 50-year-old female with a history of diabetes mellitus, who complained of swelling and pain in the posterior cervical area. Inadequate incision was performed in regional hospital and visited our institution for definitive treatment. Diagnosis of necrotizing fasciitis was confirmed by the findings during the surgery and histological report. (a): Wound at presentation. (b): Intraoperative picture of the patient. Aggressive debridement was performed immediately. (c): Necrotic tissue remained exist following the initial debridement and second debridement was performed. (d): the incision healed completely and the skin graft took well. 